\title{
Factores ambientales asociados con la preferencia de hábitat de larvas de tricópteros en cuencas con bosque seco tropical (Tolima, Colombia)
}

\author{
Jesús M. Vásquez-Ramos ${ }^{1}$, Giovany Guevara-Cardona², Gladys Reinoso-Flórez ${ }^{3}$
}

1. Grupo de Investigación en Zoología (GIZ), Facultad de Ciencias, Universidad del Tolima, Ibagué, Tolima, Colombia. Programa de Biología, Facultad de Ciencias Básicas e Ingeniería, Universidad de los Llanos, Villavicencio, Colombia; jvasquez@unillanos.edu.co

2. Departamento de Desarrollo Rural y Recursos Naturales, Facultad de Ciencias Agropecuarias, Universidad de Caldas, Manizales, Caldas, Colombia; ggcolombia@gmail.com

3. Grupo de Investigación en Zoología (GIZ), Facultad de Ciencias, Universidad del Tolima, Ibagué, Tolima, Colombia; greinoso@ut.edu.co

Recibido 12-XII-2013. Corregido 20-I-2014. Aceptado 13-II-2014.

\begin{abstract}
Environmental factors associated with habitat preferences by caddisfly larvae in tropical dry forest watersheds (Tolima, Colombia). River ecosystems, mainly those draining tropical dry forests, are among the most endangered tropical ecosystems and a major conservation priority in South America, as elsewhere. In this study, we assessed the influence of environmental factors (e.g., precipitation) and riparian vegetation on Trichoptera larval assemblages colonizing four substrates (rock, gravel, sand, and litter) in the Venadillo and Opia watersheds (Tolima, Colombia). In each river, five $20 \mathrm{~m}$ reaches nested into two $100 \mathrm{~m}$ segments (one at $\sim 550$ and another at $\sim 250 \mathrm{masl}$ ), were surveyed for benthic invertebrates in the above mentioned substrates. In addition, water samples were collected for physicochemical analyses and the QBR index ("qualitat del bosc de ribera" or riparian forest quality) was applied in both rivers. A total of 6282 larvae were collected, belonging to 11 families and 22 genera, representing $73.30 \%$ and $43.13 \%$ of the Trichoptera fauna reported to Colombia, respectively. The most abundant families were Hydropsychidae $(49.86 \%)$ and Philopotamidae $(25.44 \%)$ and the least abundant Odontoceridae $(0.16 \%)$ and Hydrobiosidae $(0.06 \%)$. The genera Smicridea, Chimarra, Protoptila, Neotrichia, and Leptonema, were common during dry and rainy seasons. The main factors related to changes in composition, richness, and abundance of larval Trichoptera were seasonality and riparian vegetation, which can influence organic matter supply, availability and stability of substrates, and colonization and population dynamics. Trichoptera assemblages showed no significant differences among substrates. However sampling points located at high elevation and in non-urbanized areas offered the largest variety of substrates and richness. Our results indicate that Trichoptera larvae are an important biotic element in freshwater ecosystems and that they are sensitive to environmental changes. Hence, our study suggests that caddisflies may be used as potential organisms for the biomonitoring of tropical dry forest rivers. The implementation of these studies is urgent, considering that degradation of freshwater ecosystems tends to be severe and persistent in dry forest. Rev. Biol. Trop. 62 (Suppl. 2): 21-40. Epub 2014 April 01.
\end{abstract}

Key words: aquatic insects, Trichoptera, tropical dry forest, freshwater ecosystem, precipitation, benthic substrates.

Los ecosistemas lóticos (ríos y quebradas) experimentan variaciones espaciales y temporales tanto en sus características físicas y químicas como en sus comunidades biológicas, razón por la cual se han constituido en temas centrales de investigación ecológica, particularmente durante las últimas décadas (Allan, 2004; Latrubesse, Stevaux \& Sinha, 2005;
Johnson \& Host, 2010; Winemiller, Flecker \& Hoeinghaus, 2010). Los ríos son elementos fundamentales en la integración del paisaje, suministran muchos bienes y servicios ambientales de alto valor para la biota y la humanidad, y constituyen factores claves para el desarrollo de la sociedad y la supervivencia de las especies (Allan, 2004; Roldán \& Ramírez, 2008). 
No obstante, muchos de estos sistemas acuáticos y sus zonas ribereñas, son afectados por diferentes actividades antropogénicas que amenazan su permanencia a largo plazo (Dudgeon, et al., 2006).

A escala de cuenca hidrográfica existen numerosos impactos de mayor o menor complejidad, que condicionan la diversidad biótica en ríos y quebradas (Dudgeon et al., 2006; Strayer, 2006; Chará, Pedraza, Giraldo \& Hincapié, 2007; Colwell \& Hix, 2008; Death \& Collier, 2009; Encalada, Calles, Ferreira, Canhoto \& Graça, 2010). Por ejemplo, aquellos asociados con la vegetación ribereña, que es el hábitat biofísico más diverso, dinámico y complejo en la interfaz entre el ecosistema terrestre y acuático, y que soporta agudos gradientes ambientales, procesos ecológicos y diversas comunidades (Naiman, Decamps \& Pollock, 1993). Las funciones de la vegetación ribereña incluyen la regulación de la temperatura y escorrentía superficial y el aporte de materia orgánica gruesa y fina, que es aprovechada como sustrato por organismos bentónicos, para su colonización, alimentación o confección de refugios (Naiman, Decamps \& Pollock, 2005; Spies, Froehlich \& Kotzian, 2006). Sin embargo, los efectos de la cobertura ribereña sobre los organismos acuáticos pueden diferir entre regiones (Utz, Hilderbrand \& Boward, 2009), dado que los sistemas lóticos cambian comparativamente en forma y función a causa de las variaciones en topografía, geología y características climáticas (Poff \& Zimmerman, 2010).

El bosque seco tropical está enmarcado por promedios de temperatura superiores a $24^{\circ} \mathrm{C}$, precipitación total anual entre 800 y 2 $000 \mathrm{~mm}$ y relaciones de evapotranspiración potencial entre 0.8 y 2.0 , que se presentan en el piso térmico cálido, en altitudes que van desde el nivel del mar hasta los $1000 \mathrm{~m}$ de altitud (Díaz, 2006; Holdridge, 1987). En la configuración de las áreas de distribución actual de los bosques secos tropicales, el ser humano ha desempeñado un papel importante al preferir asentarse en regiones secas, con disponibilidad de agua corriente y mayor fertilidad de los suelos para agricultura (Díaz, 2006; Murphy \&
Lugo, 1986). Como consecuencia, vastas áreas originalmente cubiertas por bosques caducifolios en Asia, África y América han sido convertidas en extensos campos de cultivos irrigados (Chazdon, 2003), disminuyendo la diversidad y riqueza de plantas y animales (Chazdon, 2003; Dunn, 2004). En Mesoamérica, por ejemplo, la cobertura actual de bosques secos es inferior al $2 \%$ de la original (Janzen, 1988). En Colombia, es considerado uno de los ecosistemas más degradados, fragmentados y menos conocidos; la mayoría de remanentes se localizan en áreas de intenso uso ganadero y agrícola, como es el caso de la región Caribe y de los valles interandinos drenados por los ríos Cauca y Magdalena (CORTOLIMA, 1998; IAvH, 1998). Se estima que del total de los bosques secos del valle del Magdalena, persisten menos de 5 000ha en zonas altamente fragmentadas (IAvH, 1998). El departamento del Tolima concentra parte de estos parches de extensión variable (entre 50 y $200 \mathrm{ha}$ ), principalmente en la parte norte, mientras otros fragmentos ribereños y aislados ( $\leq 1 \mathrm{ha}$ ), se encuentran distribuidos en los departamentos de Cundinamarca, Caldas y Huila (IAvH, 1998). A pesar de que es menos diverso y la productividad neta anual de biomasa es menor comparado con el bosque húmedo, posee mayor diversidad de formas vegetales y animales que configuran las propiedades biológicas y ecosistémicas de éstos (Barreda-Bautista, López-Caloca, Couturier \& Silván-Cárdenas, 2011). Por todo lo anterior, el bosque seco es considerado uno de los ecosistemas terrestres más amenazados del mundo, donde la escasa investigación sobre éste y sus procesos sucesionales no ha permitido el desarrollo y la implementación de acciones de conservación apropiadas (Bianchi \& Haig, 2013; Quesada et al., 2009).

Los estudios sobre la fauna de macroinvertebrados de ríos tropicales colombianos son escasos (Zúñiga, Rojas \& Serrato, 1994; AriasDíaz, Reinoso, Guevara \& Villa, 2007; Chará et al., 2007; Reinoso, Guevara, Arias \& Villa, 2007a; Reinoso, Guevara, Vejarano, García \& Villa, 2007b; Vásquez-Ramos \& Reinoso, 2012), en su mayoría están orientados a evaluar 
la composición y estructura de esta biota y sus posibles relaciones con las condiciones fisicoquímicas del agua. Otros resaltan el papel bioindicador de los macroinvertebrados acuáticos (Posada, Roldán \& Ramírez, 2000; Reinoso et al., 2007b; Roldán \& Ramírez, 2008), entre los que se destacan los tricópteros. Estos estudios resaltan cómo su riqueza está principalmente asociada con la diversidad de sustratos presentes en un área determinada, de tal manera que las alteraciones antropogénicas en la composición física y química del agua, se reflejan en diversos atributos ecológicos de estos organismos (Rincón, 1996; Ballesteros, Zúniga \& Rojas-Hernández, 1997; Posada-Garcia \& Roldán, 2003; Muñoz-Quesada, 2004; Guevara, Reinoso \& Villa, 2005; Springer, 2006; Spies et al., 2006; Vásquez-Ramos, Ramírez-Díaz, Reinoso \& Guevara, 2010).

El objetivo del presente estudio fue determinar los principales factores que influyen en el establecimiento de los ensamblajes de larvas del orden Trichoptera en cuatro sustratos (roca, grava, arena y hojarasca) presentes en dos cuencas con bosque seco, ubicadas en el departamento del Tolima (región central de Colombia). Lo anterior se realizó durante un ciclo hidrológico, teniendo en cuenta algunos parámetros físicoquímicos y el uso del suelo (agricultura y urbanización). Se espera que esta información constituya una base fundamental para la elaboración e implementación de acciones de monitoreo y preservación de la fauna acuática y bosques ribereños en cuencas Neotropicales.

\section{MATERIALES Y MÉTODOS}

Área de estudio: Las cuencas se localizan en la zona de vida de bosque seco tropical (CORTOLIMA, 1998), e incluyó los ríos Venadillo y Opia. El río Venadillo tiene un área de $188.4 \mathrm{~km}^{2}$, un cauce que recorre $44.9 \mathrm{~km}$, con una pendiente media de $4.5 \%$; se origina a $2250 \mathrm{msnm}$ y desemboca en el río Magdalena a 210msnm (Andrade \& Lozano, 1986). La cuenca del río Opia cuenta con un área aproximada de $325 \mathrm{~km}^{2}$ y una longitud de $65 \mathrm{~km}$, nace a $1038 \mathrm{msnm}$ en el Municipio de Ibagué y desemboca en el río Magdalena a 240msnm. Presenta una pendiente media de $18.6 \%$ (Castañeda, Medina, Méndez \& Quimbayo, 1989; Cuadro 1). En cada río, se seleccionaron dos tramos de $100 \mathrm{~m}$, ubicados a $\sim 550 \mathrm{y} \sim 250 \mathrm{msnm}$ (Cuadro 1, Fig. 1) y se tomaron muestras fisicoquímicas y biológicas bimensuales durante un año (entre febrero de 2011 y enero de 2012) en los periodos de alta y baja precipitación,

\section{CUADRO 1}

Información sobre la ubicación de los tramos seleccionados en los ríos Venadillo y Opia, afluentes del Río Magdalena, zona central de Colombia

TABLE 1

Information on the study reach location in the Venadillo and Opia rivers, tributaries of the Magdalena River, central part of Colombia

\begin{tabular}{|c|c|c|c|c|c|}
\hline \multirow{2}{*}{ Localidad } & & \multirow{2}{*}{ Municipio } & \multicolumn{2}{|c|}{ Coordenadas } & \multirow{2}{*}{$\begin{array}{l}\text { Elevación } \\
\text { (m) }\end{array}$} \\
\hline & & & $\mathrm{N}$ & $\mathrm{O}$ & \\
\hline $\begin{array}{l}\text { R. Venadillo-El Palmar } \\
(\mathrm{RVpa}) *\end{array}$ & $\begin{array}{l}\text { Zona aguas arriba del casco urbano } \\
\text { del municipio de Venadillo }\end{array}$ & Venadillo & $4^{\circ} 46^{\prime} 19.3^{\prime \prime}$ & $74^{\circ} 57^{\prime} 26.4^{\prime \prime}$ & 570 \\
\hline $\begin{array}{l}\text { R. Venadillo-Vía Ambalema } \\
\text { (RVam)* }\end{array}$ & $\begin{array}{l}\text { Zona aguas abajo del casco urbano } \\
\text { del municipio de Venadillo }\end{array}$ & Ambalema & $4^{\circ} 43^{\prime} 36.7^{\prime \prime}$ & $74^{\circ} 50^{\prime} 54.4^{\prime \prime}$ & 259 \\
\hline $\begin{array}{l}\text { R. Opia-El Tambor } \\
\text { (ROta)* }\end{array}$ & $\begin{array}{l}\text { Zona aguas arriba del casco urbano } \\
\text { del municipio de Piedras }\end{array}$ & Piedras & $4^{\circ} 27^{\prime} 30.2^{\prime \prime}$ & $74^{\circ} 57^{\prime} 35.0^{\prime \prime}$ & 559 \\
\hline $\begin{array}{l}\text { R. Opia-El Guadual } \\
\text { (ROgu)* }\end{array}$ & $\begin{array}{l}\text { Zona aguas abajo del casco urbano } \\
\text { del municipio de Piedras. }\end{array}$ & Piedras & $4^{\circ} 33^{\prime} 40.1^{\prime \prime}$ & $74^{\circ} 50^{\prime} 26.6^{\prime \prime}$ & 269 \\
\hline
\end{tabular}

(*) Abreviaturas empleadas para cada uno de los tramos evaluados de los ríos Venadillo y Opia. 


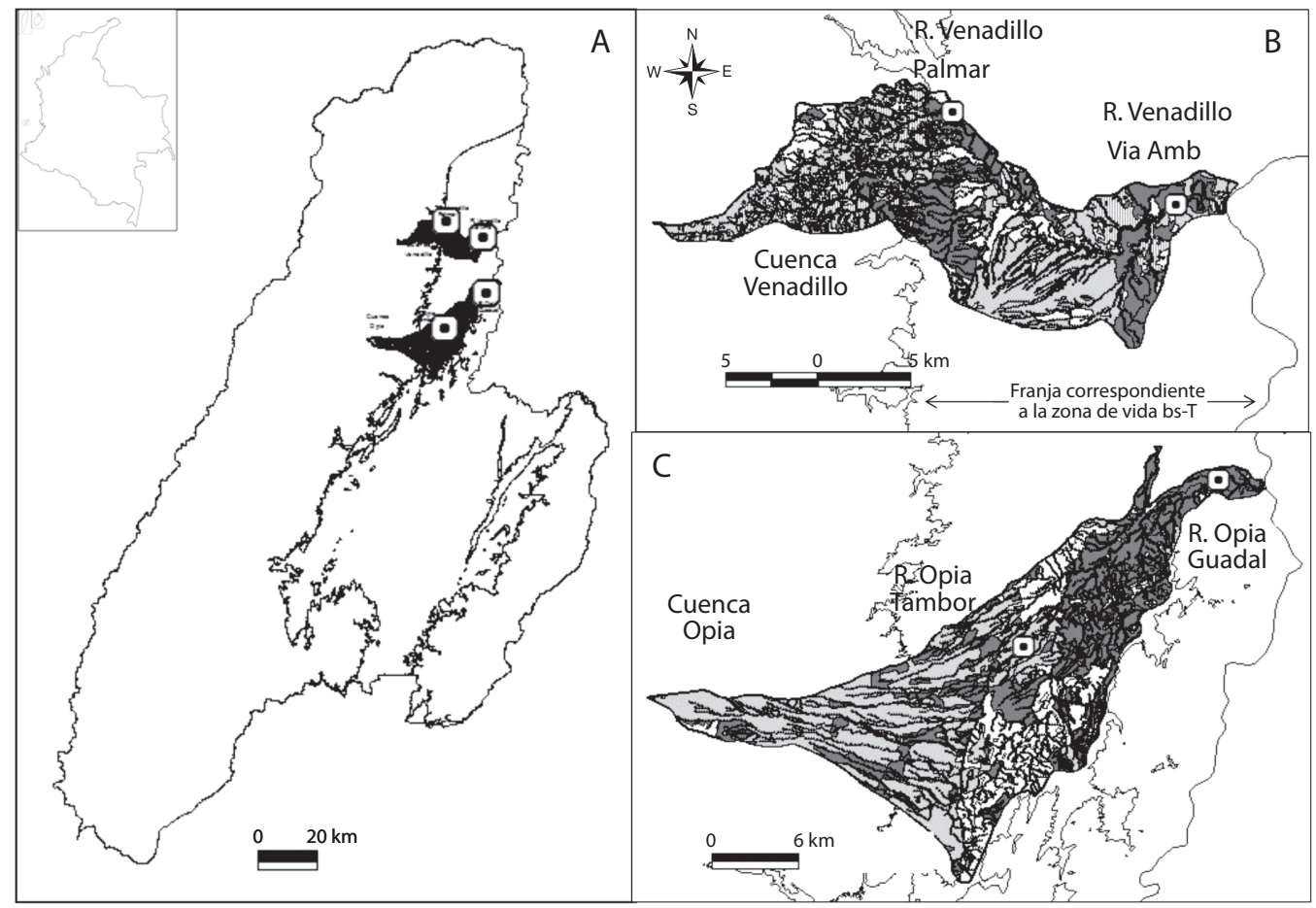

Fig. 1. Área de estudio con las cuencas y tramos evaluados en los ríos Venadillo y Opia (Tolima, Colombia). Se indican los usos del suelo predominantes en cada una de las cuencas.

Fig. 1. Study area with the watersheds and reaches evaluated in the Venadillo and Opia rivers (Tolima, Colombia). Predominant land uses in each watershed are also indicated.

teniendo en cuenta los registros pluviométricos históricos ( 10 años) (Fig. 2).

Recolecta de larvas de tricópteros. Cada uno de los tramos evaluados $(100 \mathrm{~m})$ se dividieron en cinco secciones $(20 \mathrm{~m})$, en las que se tomaron hasta cuatro muestras con una red Surber $(30 \times 30 \mathrm{~cm}, 250 \mu \mathrm{m}$ de abertura de malla) de acuerdo con la disponibilidad de hábitat (roca, grava, arena, hojarasca), para un máximo de 240 muestras en cada río $(=2$ tramos x 5 secciones $\times 4$ muestras $\times 6$ colectas bimensuales). En campo, las muestras se fijaron con formol (10\%), y posterior a la limpieza y separación de los organismos, se almacenaron en etanol (70\%). Los organismos se determinaron a nivel de género con diferentes claves y descripciones (Angrisano, 1995; Angrisano \& Korob, 2001; Posada-Garcia \& Roldán, 2003; Oliveira \& Hamada, 2004; Oliveira, Hamada \&
Nessimian, 2005; Springer, 2006; Angrisano \& Sganga, 2009; Domínguez \& Fernández, 2009) y se ingresaron a la Colección Zoológica de la Universidad del Tolima (CZUT-Ma).

Variables físicas, químicas y bacteriológicas. En cada sitio y fecha de muestreo se determinó in situ la temperatura $\left({ }^{\circ} \mathrm{C}\right) \mathrm{y}$ conductividad $\left(\mu \mathrm{Scm}^{-1}\right)$ del agua con un equipo portátil SCHOTT Handylab multi12/Set. Asimismo, se determinó el caudal utilizando el área transversal de la corriente, la profundidad media y la velocidad de flujo de un objeto flotante (Roldán \& Ramírez, 2008). En laboratorio se determinaron los parámetros de turbiedad (UNT), $\mathrm{pH}$, nitratos $\left(\mathrm{NO}_{3}{ }^{-}\right.$; $\left.\mathrm{mgL}^{-1}\right)$, fosfatos $\left(\mathrm{PO}_{4}^{-} ; \mathrm{mgL}^{-1}\right)$, sólidos totales $\left(\mathrm{mgL}^{-1}\right), \mathrm{DBO}_{5}\left(\mathrm{mg} \mathrm{O}_{2} \mathrm{~L}^{-1}\right), \%$ de saturación de oxígeno y oxígeno disuelto $\left(\mathrm{mg} \mathrm{O}_{2} \mathrm{~L}^{-1}\right)$, y parámetros bacteriológicos como Escherichia 


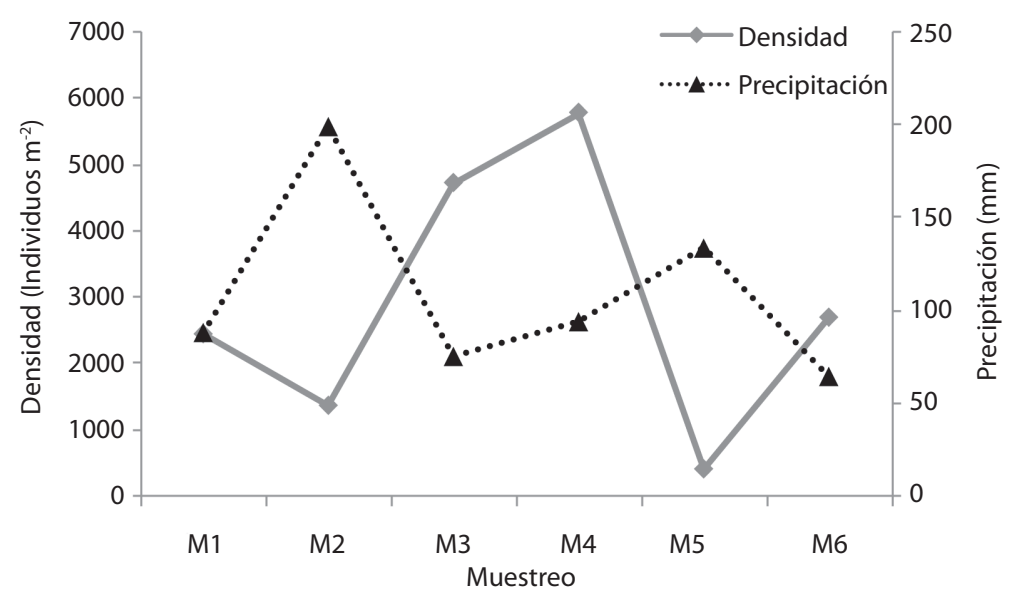

Fig. 2. Densidad total y precipitación media mensual (2001-2010) en las cuencas de los ríos Venadillo y Opia, durante seis muestreos realizados.

Fig. 2. Total density and average monthly rainfall (2001-2010) in the Venadillo and Opia river basins, during six realized samplings.

coli y coliformes totales. También se aplicó el índice QBR (Munné, Prat, Solà, Bonada \& Rieradevall, 2003) para la determinación de la calidad de la vegetación ribereña en cada punto de muestreo seleccionado. El QBR se basa en la evaluación de cuatro componentes del hábitat ribereño: cobertura total de la vegetación ribereña, estructura y calidad de la cobertura, y alteraciones del canal (Jáimez-Cuéllar et al., 2002; Munné et al., 2003). Para el componente de calidad de la cobertura, se elaboró una lista de las especies de árboles y arbustos nativos y no nativos para cada tramo evaluado (Colwell \& Hix, 2008; Munné et al., 2003).

Análisis de datos. Para determinar las variaciones en la densidad total y por género (individuos $\mathrm{m}^{-2}$ ) a nivel de muestreo (M1 $\mathrm{M} 6$; donde $\mathrm{M}=$ muestreo, siguiendo un orden cronológico o periodo de recolecta), localidad (RVpa, RVam, ROta, ROgu) y sustrato (roca, grava, arena y hojarasca), se empleó la prueba no paramétrica de Kruskal-Wallis, y representación visual mediante diagramas de caja y bigotes (box plots). La densidad total por muestreo se comparó con los registros de precipitación promedio anual/local de los últimos diez años, para determinar la influencia de patrones estacionales sobre la variación temporal en la abundancia de tricópteros. Dado que no todos los sustratos fueron registrados en los tramos evaluados en los diferentes periodos de muestreo, se evidenciaron diferencias en el número total de individuos por sustrato. Por lo tanto, para calcular la riqueza se empleó un análisis con curvas de rarefacción para géneros, basado en los individuos presentes en sustratos y localidades. Se calculó tanto la riqueza (S) neta como la rarefaccionada (Srar), el índice de diversidad de Shannon-Wiener (H'), y el de dominancia de Simpson $(\lambda)$ con el software PAST versión 2.13 (Hammer, Harper \& Ryan, 2001). Asimismo, para comparar globalmente la comunidad de tricópteros por sustrato y tramo, se realizó una ordenación nMDS (Nonmetric multidimensional scaling), mediante transformación de los datos $(\log n+1)$ y aplicación del índice de similitud de Bray-Curtis con el software PRIMER versión 6.10 (Clark \& Gorley, 2006). Por otra parte, se realizó un Análisis de Correspondencia Canónica (ACC) utilizando CANOCO versión 4.56 (Braak \& Smilauer, 2009) para evaluar la relación entre la comunidad de tricópteros, y las variables fisicoquímicas y ambientales. Se utilizaron únicamente taxones con abundancia superior 
al $1 \%$ del total, después de la transformación mediante raíz cuadrada. Todas las variables fisicoquímicas (excepto $\mathrm{pH}$ ) y ambientales se transformaron $(\log n+1)$ previo al análisis, para reducir la asimetría en la distribución de las mismas (Leira \& Sabater, 2005); también se realizó una prueba de correlación múltiple entre todas las variables para evitar la inclusión de aquellas autocorrelacionadas.

\section{RESULTADOS}

Se registraron 6282 larvas pertenecientes a 11 familias y 22 géneros. Las familias más abundantes fueron Hydropsychidae (49.86\%) y Philopotamidae (25.44\%), seguidas por Hydroptilidae (9.87\%) y Glossosomatidae (9.84\%); y las de menor abundancia Odontoceridae $(0.16 \%)$ e Hydrobiosidae $(0.06 \%)$ (Cuadro 2). Se registró una diferencia significativa ( $\mathrm{p}=0.039$, Kruskal-Wallis) al comparar la densidad total de individuos en los diferentes muestreos (agrupados por localidad y sin discriminar sustratos); durante M2 $(\mu \pm \mathrm{DE}, 1$ $375 \pm 1081$ individuos) y M5 (419.4 \pm 159.6$)$ se registró la menor densidad, mientras que en M1

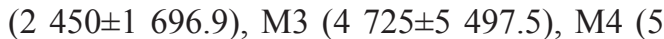

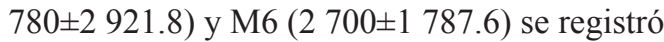
la mayor densidad de organismos (Fig. 2).

CUADRO 2

Abundancia relativa (\%) de larvas de tricópteros registradas en seis muestreos realizados en cuatro tramos de los ríos Venadillo y Opia (Tolima, Colombia)

TABLE 2

Relative abundance (\%) of caddisfly larvae registered in six sampling occasions, in four sites of the Venadillo and Opia rivers (Tolima, Colombia)

\begin{tabular}{|c|c|c|c|c|c|c|c|}
\hline \multirow{2}{*}{ Familia } & \multirow{2}{*}{ Género } & \multicolumn{6}{|c|}{ Muestreos } \\
\hline & & M1 & M2 & M3 & M4 & M5 & M6 \\
\hline Calamoceratidae & Phylloicus sp. & 2.23 & 4.58 & 0.87 & 0.00 & 0.00 & 0.00 \\
\hline Glossosomatidae & Protoptila sp. & 16.76 & 0.00 & 10.04 & 17.54 & 7.89 & 20.73 \\
\hline Helicopsychidae & Helicopsyche sp. & 1.12 & 0.76 & 2.18 & 2.24 & 0.00 & 0.00 \\
\hline Hydrobiosidae & Atopsyche sp. & 1.68 & 0.00 & 0.00 & 0.00 & 0.00 & 0.00 \\
\hline \multirow[t]{3}{*}{ Hydropsychidae } & Leptonema sp. & 12.29 & 10.69 & 10.48 & 4.85 & 10.53 & 10.37 \\
\hline & Macronema sp. & 0.00 & 0.00 & 0.00 & 0.37 & 0.00 & 0.00 \\
\hline & Smicridea sp. & 24.58 & 37.40 & 24.02 & 22.01 & 35.53 & 28.66 \\
\hline \multirow[t]{8}{*}{ Hydroptilidae } & Ceratotrichia sp.* & 0.00 & 0.00 & 0.00 & 0.37 & 0.00 & 0.00 \\
\hline & Hydroptila sp. & 0.00 & 0.00 & 6.99 & 1.12 & 0.00 & 1.83 \\
\hline & Mayatrichia sp.* & 0.00 & 0.00 & 0.00 & 0.75 & 0.00 & 0.61 \\
\hline & Neotrichia sp. & 15.08 & 20.61 & 12.23 & 15.67 & 13.16 & 6.10 \\
\hline & Ochrotrichia sp. & 0.00 & 2.29 & 0.44 & 0.00 & 0.00 & 2.44 \\
\hline & Zumatrichia sp. & 1.68 & 1.53 & 0.44 & 2.24 & 0.00 & 0.61 \\
\hline & Leucotrichia sp. & 0.00 & 0.00 & 0.44 & 0.00 & 0.00 & 0.00 \\
\hline & Hydroptilidae spp. & 3.35 & 0.00 & 0.87 & 4.48 & 5.26 & 3.05 \\
\hline \multirow[t]{3}{*}{ Leptoceridae } & Grumichella sp. & 2.23 & 3.05 & 0.00 & 0.00 & 5.26 & 6.10 \\
\hline & Nectopsyche sp. & 6.70 & 1.53 & 4.37 & 5.22 & 1.32 & 0.61 \\
\hline & Oecetis sp. & 0.00 & 0.00 & 1.31 & 1.12 & 1.32 & 0.61 \\
\hline Odontoceridae & Marilia sp. & 1.12 & 2.29 & 0.00 & 1.49 & 0.00 & 0.61 \\
\hline Philopotamidae & Chimarra sp. & 8.38 & 14.50 & 23.14 & 17.16 & 13.16 & 14.02 \\
\hline \multirow[t]{2}{*}{ Polycentropodidae } & Polycentropus sp. & 0.56 & 0.76 & 1.31 & 1.12 & 5.26 & 0.61 \\
\hline & Polyplectropus sp. & 0.00 & 0.00 & 0.00 & 0.75 & 0.00 & 0.00 \\
\hline Xiphocentronidae & Xiphocentron sp. & 2.23 & 0.00 & 0.87 & 1.49 & 1.32 & 3.05 \\
\hline
\end{tabular}

*Nuevos registros para el departamento del Tolima y Colombia. $\mathrm{M}=$ muestreos realizados. $\mathrm{M}=$ samplings. 
A nivel de género se registraron variaciones en la densidad entre localidades, muestreos y sustratos. Los análisis de Kruskal-Wallis determinaron que Helicopsyche, Hydroptila y Protoptila registraron diferencia significativa en la densidad entre muestreos y localidades (p<0.05; Fig. 3 y 4); en los muestreos M1, M3, M4 y M6 (que corresponden a periodos de menor precipitación; Fig. 2), estos organismos registraron la mayor densidad. Sin embargo, espacialmente Helicopsyche e Hydroptila registraron mayor densidad en los tramos RVpa y ROta (que no tienen influencia directa de la urbanización), y Protoptila alcanzó sus mayores densidades en los tramos de zonas bajas (RVam y ROgu). Por otra parte, Leptonema, Nectopsyche, Neotrichia, Polycentropus y Xiphocentron registraron diferencias significativas entre sustratos y localidades ( $<<0.05$; Fig. 4 y 5 ). Las mayores densidades se registraron en grava, hojarasca y roca, exceptuando Nectopsyche y Polycentropus que fueron abundantes solo en hojarasca, y Xiphocentron solo en roca. Asimismo, Leptonema, Nectopsyche, Polycentropus y Xiphocentron fueron abundantes en los tramos altos (RVpa y ROta), mientras que NeOtrichia lo fue en el río Opia y en RVam. Otros taxones registraron diferencias significativas únicamente entre localidades (Oecetis y Phylloicus; mayor densidad en RVpa), muestreos (Atopsyche; mayor densidad en M1) o sustratos (Smicridea; registró mayor abundancia en roca, grava y hojarasca) $(\mathrm{p}<0.05$; Fig. 3, 4 y 5). Se observó que Smicridea, Chimarra, Neotrichia, Leptonema, Protoptila, Helicopsyche, Hydroptila y Grumichella habitan en todos
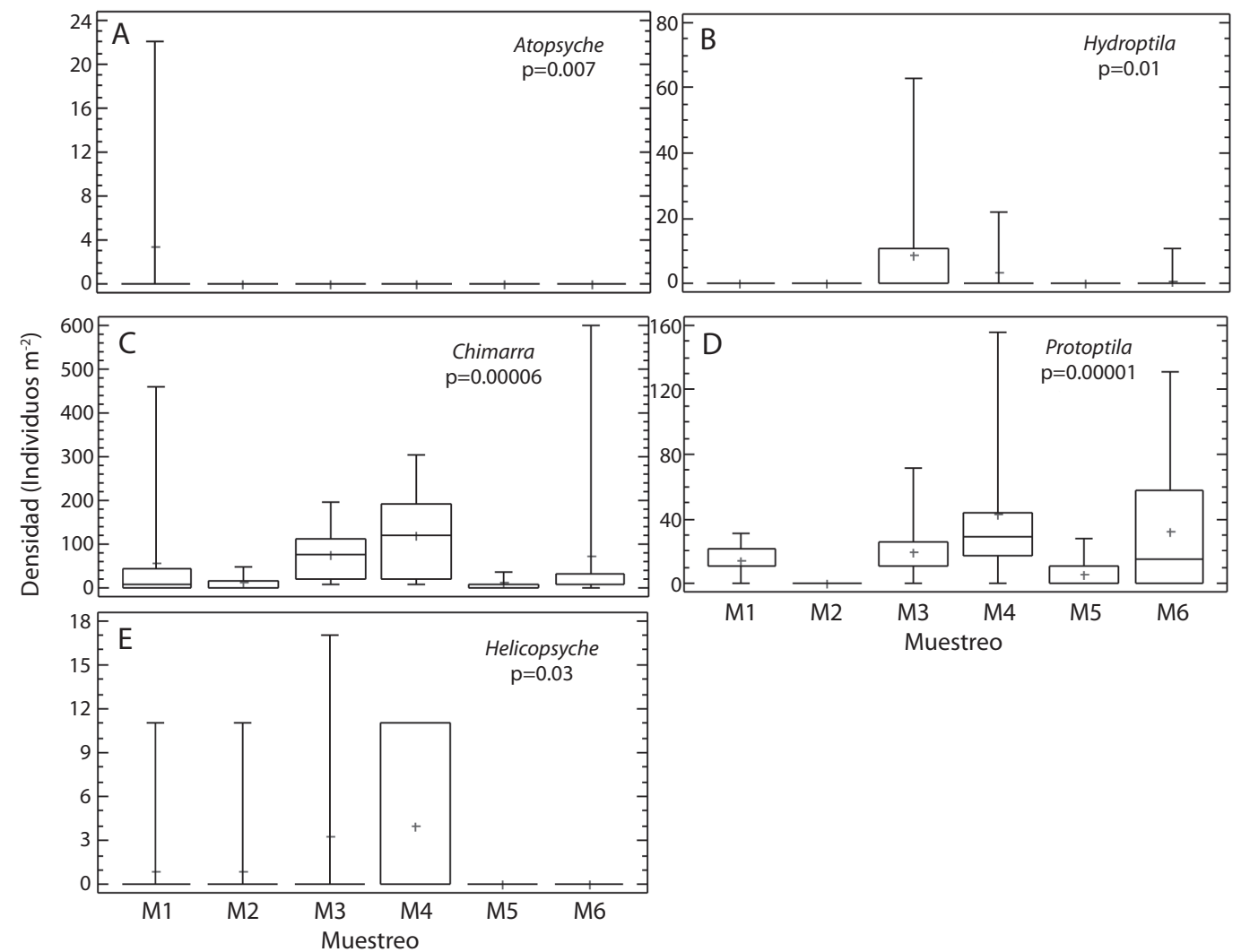

Fig. 3. Densidad media $(\mu \pm \mathrm{DE})$ por muestreo para cinco taxones en dos ríos de Colombia. Los valores p (análisis de Kruskal-Wallis) están dados para cada taxón.

Fig. 3. Mean density $(\mu \pm$ SD) per sampling for five taxa at two Colombian rivers, p values (Kruskal-Wallis'test) are indicated for each taxon. 

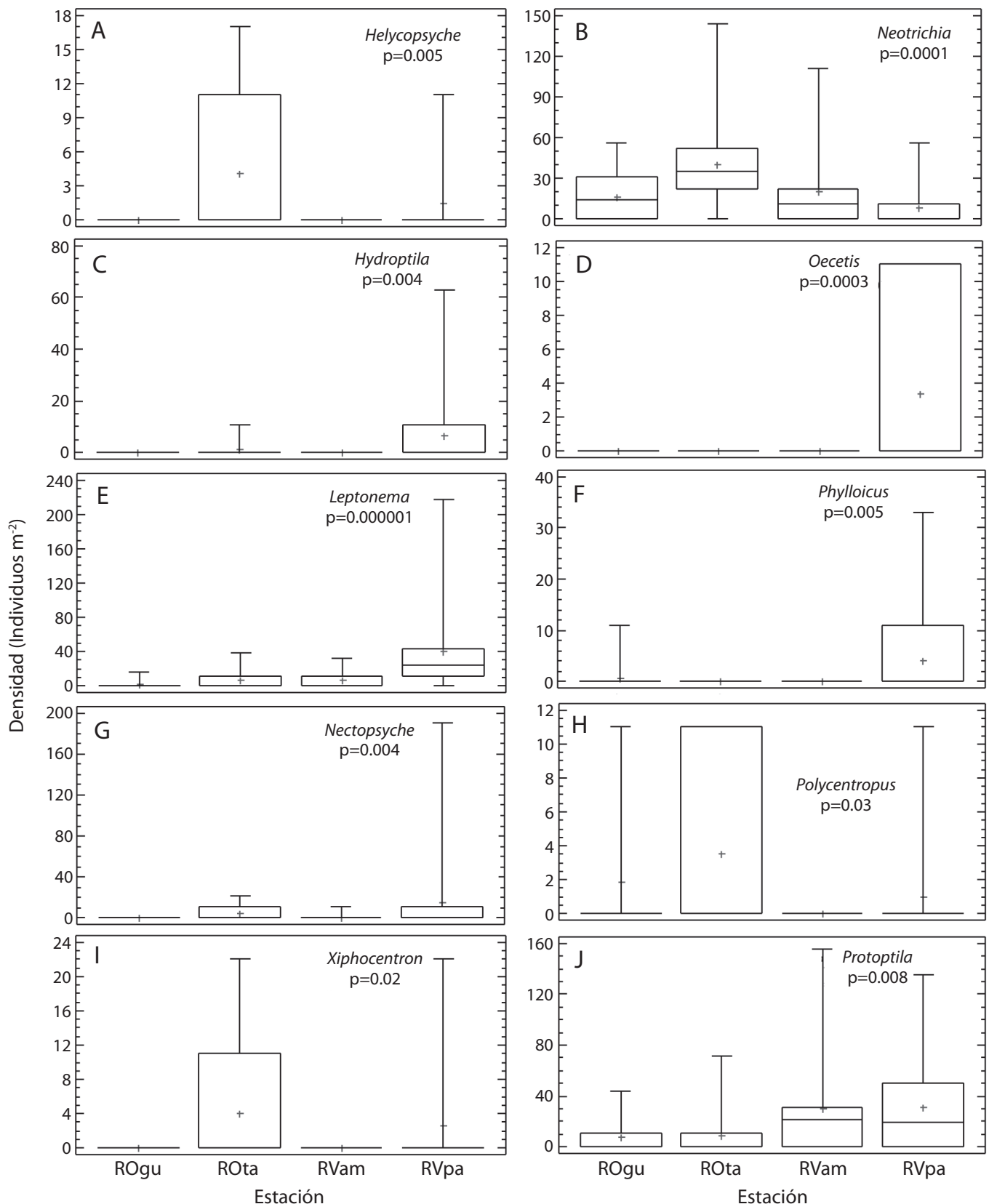

Fig. 4. Densidad media $(\mu \pm \mathrm{DE})$ por muestreo para diez taxones en dos ríos de Colombia. Los valores p (análisis de KruskalWallis) están dados para cada taxón.

Fig. 4. Mean density $(\mu \pm \mathrm{SD})$ per sampling for ten taxa at two Colombian rivers, $\mathrm{p}$ values (Kruskal-Wallis'test) are indicated for each taxon. 

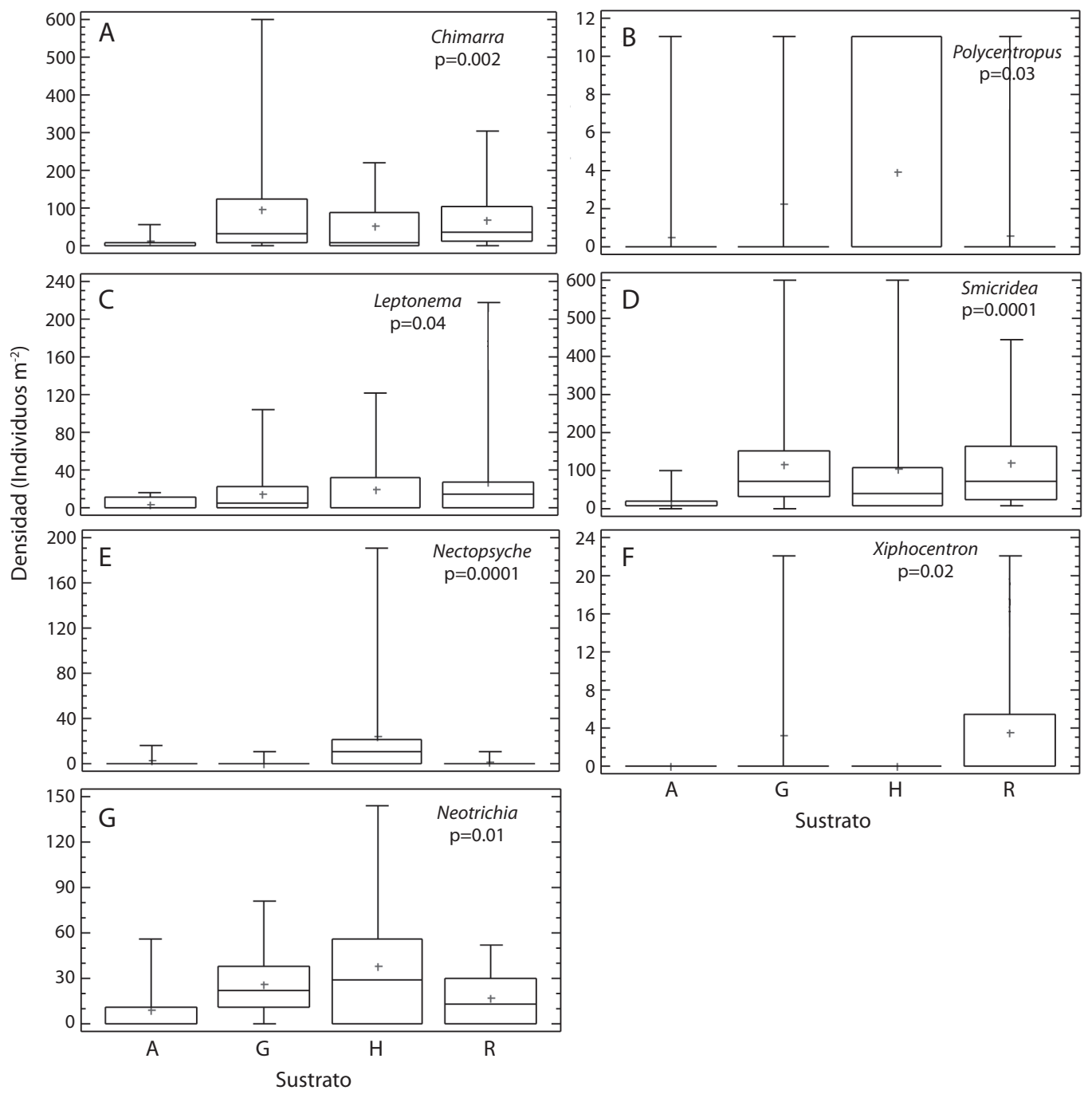

Fig. 5. Densidad media ( $\mu \pm \mathrm{DE}$ ) por muestreo para siete taxones en dos ríos de Colombia. Los valores p (análisis de Kruskal-Wallis) están dados para cada taxón.

Fig. 5. Mean density $(\mu \pm$ SD) per sampling for seven taxa at two Colombian rivers, $p$ values (Kruskal-Wallis'test) are indicated for each taxon.

los sustratos (con mayor frecuencia en grava y roca, Fig. 6), siendo los cinco primeros los de mayor frecuencia de ocurrencia. Asimismo, Xiphocentron se registró únicamente en roca y grava, Marilia en todos los sustratos excepto en hojarasca y Phylloicus en todos excepto en arena (Fig. 6).

Al agrupar todos los muestreos y realizar comparaciones por localidad y sustrato, la diversidad y riqueza más altas $(\mathrm{S}>8, \mathrm{Srar}>3$, $H^{\prime}>1.3$ ) se registraron en RVpa y ROta (Fig. 7). Temporalmente a escala de sustrato se encontró una mayor riqueza en roca y grava, mientras que la diversidad mostró un patrón inverso: arena y hojarasca registraron valores más altos (Fig. 8). Asimismo, al comparar la diversidad tanto espacial como temporalmente, se observó que los tramos RVpa y ROta exhibieron mayor 


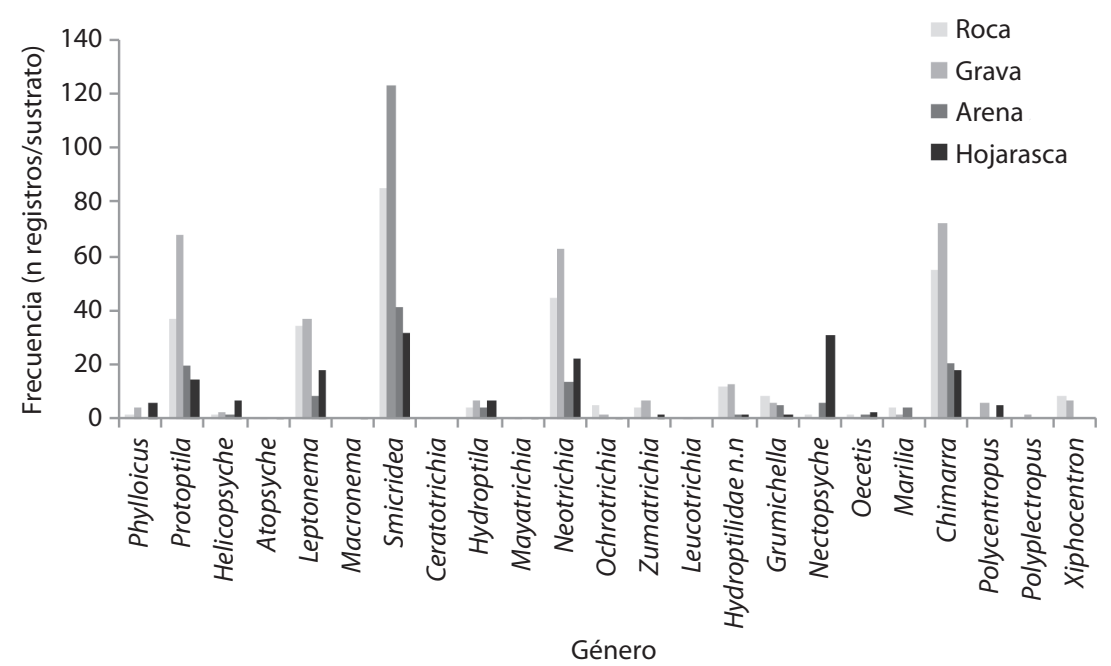

Fig. 6. Frecuencia de ocurrencia de géneros de Trichoptera por sustrato durante el periodo de estudio. Fig. 6. Total frequency of occurrence of Trichoptera genera per substrate during the sampling period.

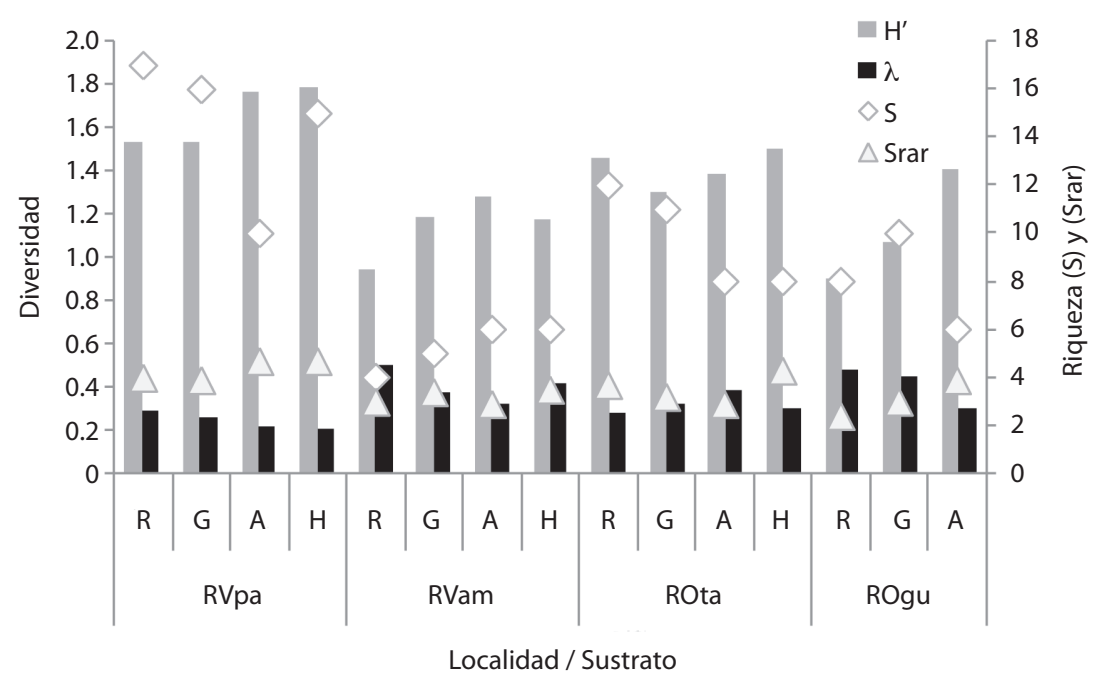

Fig. 7. Valores de diversidad de Trichoptera registrados por tramo y sustrato (hábitat) en dos ríos del departamento del Tolima. Roca (R), Grava (G), Arena (A), Hojarasca (H). Las abreviaturas de los tramos son indicadas en el Cuadro 1.

Fig. 7. Diversity values of the Trichoptera registered per reach and substrate in two rivers of the department of Tolima. Rock (R), gravel/pebble (G), sand (A), and leaflitter (H). Reach abbreviations as in Table 1.

diversidad y riqueza durante cuatro de los seis muestreos, mostrando una amplia variación a escala de sustrato (Fig. 8).

La ordenación nMDS permitió evidenciar dos grupos de larvas de tricópteros diferenciados por rango altitudinal en ambos ríos: tramos altos (RVpa y ROta) versus tramos bajos (RVam y ROgu, Fig. 9a). Mientras que a nivel de sustratos no se detectaron diferencias en los ensamblajes (Fig. 9b). Con respecto a las variables fisicoquímicas y ambientales, el río Venadillo presentó valores más bajos de $\mathrm{pH}$, conductividad, fosfatos, turbidez, QBR y profundidad, y valores más altos de caudal 


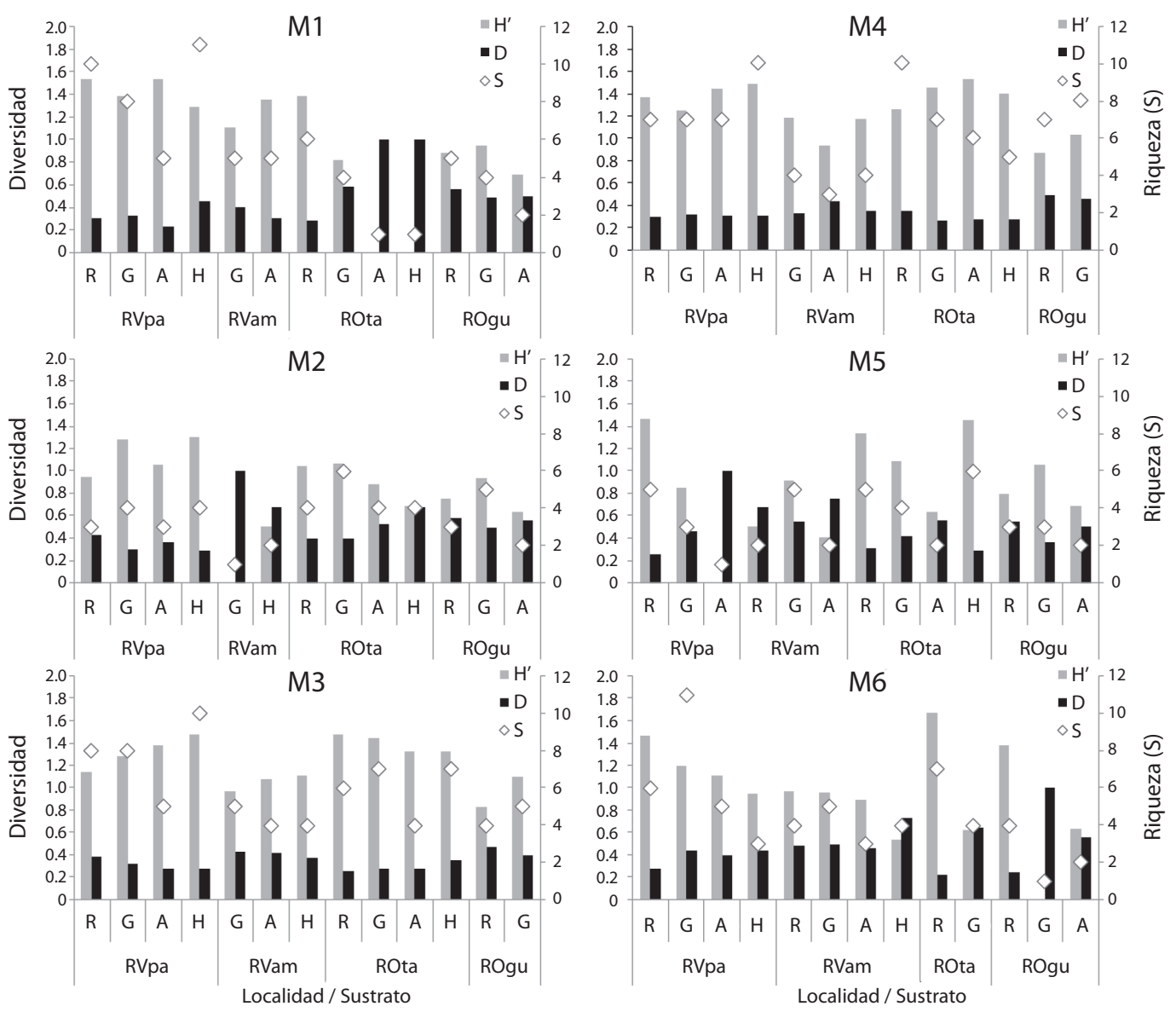

Fig. 8. Valores de diversidad de Trichoptera registrados por sustrato (hábitat) en cada tramo y muestreo, en dos ríos tropicales del departamento del Tolima. $\mathrm{M}=$ muestreos en orden cronológico. Sustratos como Fig. 7 y abreviaturas de tramos como en Cuadro 1.

Fig. 8. Diversity values of the Trichoptera registered per substrate, reach and sampling in two tropical rivers of the department of Tolima. $\mathrm{M}=$ sampling occasions. Substrates as in Fig. 7 and reach abbreviations as in Table 1.

(Cuadro 3). En el ACC, el primer ( $\mathrm{F}=2.87$, $\mathrm{p}=0.01)$ y segundo eje $(\mathrm{F}=1.87, \mathrm{p}<0.01)$ fueron significativos, explicaron el $39 \%$ de la varianza de los datos (factor $1=0.26$; factor $2=0.13$ ), donde las variables: $\mathrm{QBR}(\mathrm{p}=0.01)$, coliformes totales $(\mathrm{CT}, \mathrm{p}=0.04)$, profundidad $(\mathrm{Pr}, \mathrm{p}=0.03$ ) y conductividad $(\mathrm{CE}, \mathrm{p}=0.05)$ mostraron una relación significativa con la composición taxonómica de tricópteros. Lo observado se reflejó como sigue: a) Se visualizaron dos grupos claramente diferenciados de las localidades correspondientes al río Venadillo y al río Opia; b) Chimarra, Zumatrichia, Protoptila, Mayatrichia y Smicridea se correlacionaron con valores altos de temperatura, $\mathrm{CE}$, Pr y mayores valores del QBR que caracterizaron a los tramos bajos de ambos ríos (RVam y ROgu); y c) Phylloicus, Grumichella, Marilia, Oecetis y Nectopsyche, se correlacionaron principalmente con el tramo RVpa, donde se registraron los valores más bajos del índice QBR (Fig. 10).

\section{DISCUSIÓN}

El bosque seco tropical comprende cerca del 40\% de las áreas boscosas tropicales del mundo (Barreda-Bautista et al., 2002). Sin 
CUADRO 3

Caracterización fisicoquímica, ambiental e hidrológica de los ríos Venadillo y Opia

TABLE 3

Physicochemical, environmental and hydrological characterization of the Venadillo and Opia rivers

\begin{tabular}{|c|c|c|c|c|c|c|c|c|}
\hline & \multicolumn{4}{|c|}{ Río Venadillo } & \multicolumn{4}{|c|}{ Río Opia } \\
\hline & \multicolumn{2}{|c|}{ RVpa } & \multicolumn{2}{|c|}{ RVam } & \multicolumn{2}{|c|}{ ROta } & \multicolumn{2}{|c|}{$\mathrm{ROgu}$} \\
\hline & $\mu$ & $\mathrm{DE}$ & $\mu$ & $\mathrm{DE}$ & $\mu$ & $\mathrm{DE}$ & $\mu$ & $\mathrm{DE}$ \\
\hline Temperatura del agua $\left({ }^{\circ} \mathrm{C}\right)$ & 23.14 & $(1.20)$ & 28.97 & $(2.60)$ & 27.28 & $(0.66)$ & 27.50 & $(1.26)$ \\
\hline $\mathrm{pH}$ & 7.66 & $(0.20)$ & 7.86 & $(0.19)$ & 7.96 & $(0.10)$ & 8.05 & $(0.22)$ \\
\hline $\mathrm{O}_{2}$ disuelto $\left(\mathrm{mg} \mathrm{l}^{-1}\right)$ & 8.23 & $(2.66)$ & 7.82 & $(2.24)$ & 7.36 & $(1.90)$ & 7.47 & $(1.92)$ \\
\hline Conductividad $\left(\mu \mathrm{S} \mathrm{cm}^{-1}\right)$ & 155.25 & $(38.42)$ & 203.50 & (18.38) & 391.50 & (59.79) & 409.75 & $(65.55)$ \\
\hline Turbidez (NTU) & 14.50 & $(17.05)$ & 22.00 & $(15.39)$ & 50.83 & $(52.30)$ & 47.33 & $(34.63)$ \\
\hline Sólidos suspendidos $\left(\mathrm{mg} \mathrm{l}^{-1}\right)$ & 82.83 & $(70.91)$ & 107.33 & $(85.71)$ & 60.64 & $(61.85)$ & 77.64 & $(58.58)$ \\
\hline $\mathrm{DBO}_{5}\left(\mathrm{mg} \mathrm{l}^{-1}\right)$ & 4.77 & $(4.80)$ & 5.70 & $(5.31)$ & 6.03 & $(4.60)$ & 6.55 & $(4.84)$ \\
\hline Nitratos $\left(\mathrm{NO}_{3}^{-}\right)$ & 1.23 & $(0.64)$ & 1.81 & $(0.62)$ & 3.40 & $(1.91)$ & 2.77 & $(1.80)$ \\
\hline Fosfatos $\left(\mathrm{PO}_{4}^{-}\right)$ & 2.33 & $(1.12)$ & 4.56 & $(2.18)$ & 3.58 & $(1.74$ & 4.83 & $(4.46)$ \\
\hline Coliformes fecales & 275.33 & $(602.48)$ & 486.67 & $(382.08)$ & 345.00 & $(669.71)$ & 252.67 & (287.37) \\
\hline Coliformes totales & 99316.67 & $(240388.5)$ & 5133.33 & $(3544.9)$ & 47633.33 & (113838.8) & 9236.67 & $(17642.1)$ \\
\hline Índice QBR & 60.00 & $(0.00)$ & 85.00 & $(0.00)$ & 90.00 & $(0.00)$ & 95.00 & $(0.00)$ \\
\hline Profundidad (m) & 0.19 & $(0.07)$ & 0.30 & $(0.13)$ & 0.33 & $(0.08)$ & 0.38 & $(0.11)$ \\
\hline Cauce (m) & 16.50 & $(4.83)$ & 30.75 & $(5.47)$ & 12.28 & $(1.81)$ & 25.50 & $(2.43)$ \\
\hline Velocidad $\left(\mathrm{m} \mathrm{s}^{-1}\right)$ & 0.52 & $(0.39)$ & 0.69 & $(0.35)$ & 0.55 & $(0.31)$ & 0.49 & $(0.38$ \\
\hline Caudal $\left(\mathrm{m}^{3} \mathrm{~s}^{-1}\right)$ & 1.90 & $(1.89)$ & 7.31 & $(6.46)$ & 2.28 & $(1.74)$ & 4.60 & $(4.19)$ \\
\hline
\end{tabular}

Los datos representan la media $(\mu)$ y su respectiva desviación estándar (DE) de seis muestreos. Las abreviaturas de los tramos como en el Cuadro 1.Values indicating mean $(\mu)$ and the corresponding standard deviation (SD) of six samplings. Reach abbreviations as in Table 1.

embargo, este porcentaje global presenta una disminución paulatina como resultado de la perturbación antropogénica acelerada, principalmente durante los últimos años (Trejo \& Dirzo, 2000; Palacios-Vargas, Castaño-Meneses, Gómez-Anaya, Martínez-Yrizar, MajíaRecamier \& Martínez-Sánchez, 2007). Esto es particularmente evidente en ecosistemas dulceacuícolas y ribereños (Chará et al., 2007; Guevara et al., 2008), donde el porcentaje de conocimiento es inferior a la tasa de degradación de los mismos. De igual manera se destaca que los ecosistemas acuáticos y ribereños de zonas tropicales han sido poco estudiados y presentan muchos vacíos en investigaciones sobre densidad poblacional, respuestas a los patrones de lluvia y sequía típicos de la zona tropical, patrones de actividad, respuesta frente a variables fisicoquímicas, y efecto del sustrato sobre los ensamblajes de macroinvertebrados y particularmente de tricópteros (Domínguez \& Fernández, 2009). Los aspectos anteriores también han recibido, en general, poca atención en la dinámica faunística en ecosistemas con bosque seco, a nivel global (PalaciosVargas et al., 2007).

En nuestro estudio la mayor oferta del sustrato hojarasca se registró en los tramos ubicados aguas arriba de los centros poblados, mientras que las zonas de influencia urbana estuvieron limitadas a la oferta de arena, roca y grava. Además, la presencia de estos sustratos fue dependiente del periodo de muestreo, con menor oferta de hojarasca y arena durante los periodos más lluviosos como consecuencia del arrastre de material por el incremento del caudal y la velocidad de la corriente (VásquezRamos, obs. pers.). Las preferencias de hábitat de los macroinvertebrados bentónicos son el resultado del balance de varios requerimientos 


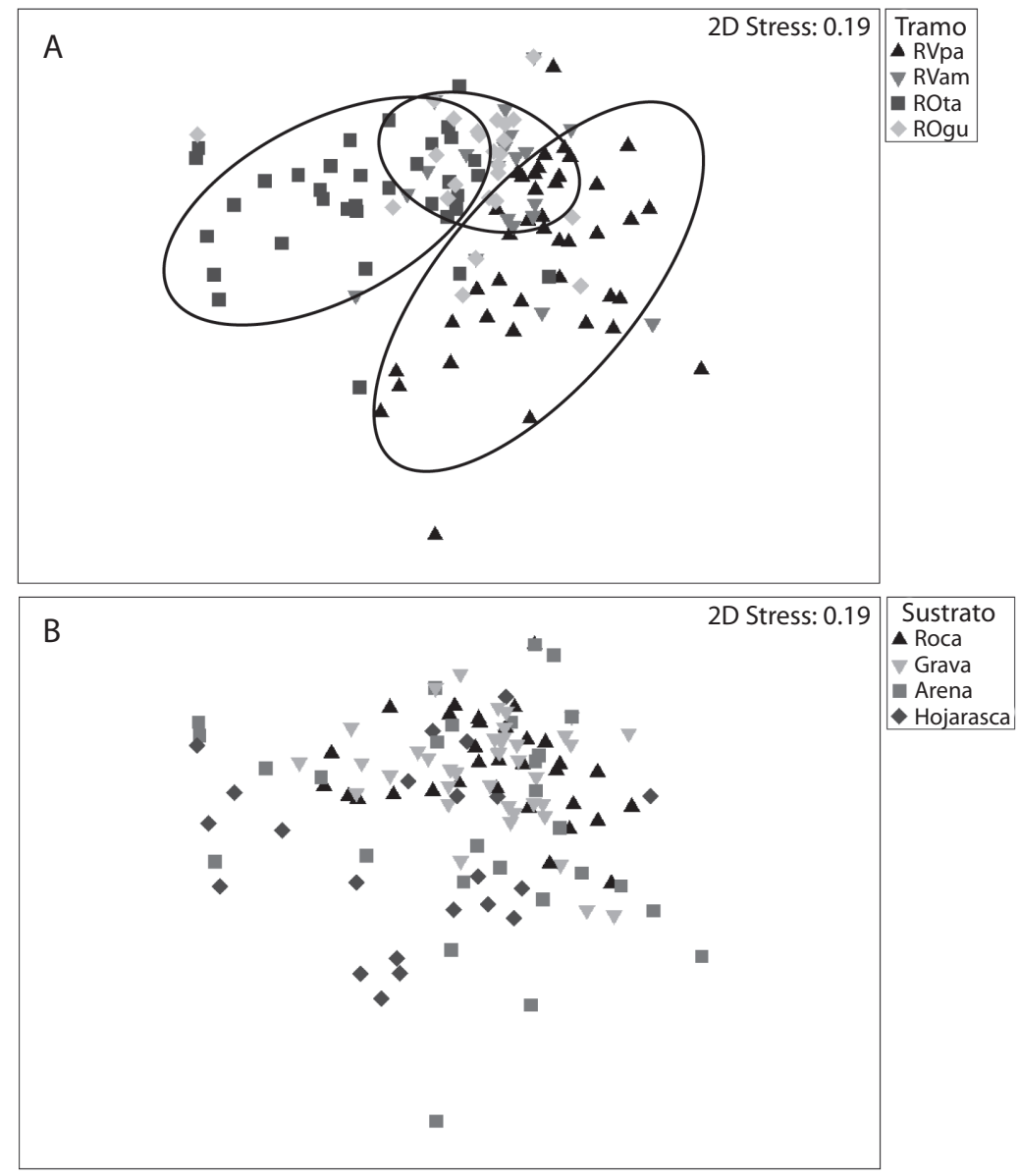

Fig. 9. Diagrama de ordenación NMDS basado en la densidad (Ind. $\mathrm{m}^{-2}$ ) de los géneros de tricópteros registrados en los seis muestreos por tramo y sustrato. Los círculos indican las agrupaciones por localidad. Las abreviaturas de tramos como en Cuadro 1.

Fig. 9. NMDS ordination based on the density (Ind. $\mathrm{m}^{-2}$ ) of caddisflies genera registered per reach and substrate in six samplings. The circles indicate the resulting groups. Reach abbreviations as in Table 1.

para su colonización y supervivencia (Negi \& Singh, 1990; Beisel, Usseglio-Polatera, Thomas \& Moreteau, 1998; Oliveira \& Nessimian, 2010). La naturaleza del sustrato junto con los patrones de caudal y la influencia directa de otras variables físicas y químicas, resultan en una distribución heterogénea de micro y mesohábitats, los cuales son colonizados por una comunidad particular de macroinvertebrados (Pardo \& Armitage, 1997). Asimismo, los hábitats para los ensamblajes de insectos acuáticos pueden ser interpretados en el marco de varias escalas espaciales y temporales (Subramanian
\& Sivaramakrishnan, 2005). Los tricópteros se han adaptado a colonizar diferentes sustratos (Holzenthal, Blahnik, Prather \& Kjer, 2007), los cuales utilizan en la confección de sus refugios, bien sea con materiales individualizados o mixtos de origen natural o antropogénico (restos de materiales inorgánicos y desechos; Guevara, 2004; Guevara et al., 2005) y para capturar alimento de una manera más eficiente (Motta \& Uieda, 2004; Angrisano \& Sganga, 2009). Algunos autores han indicado que los procesos que influyen en la comunidad de las larvas de tricópteros a escala local y regional, 


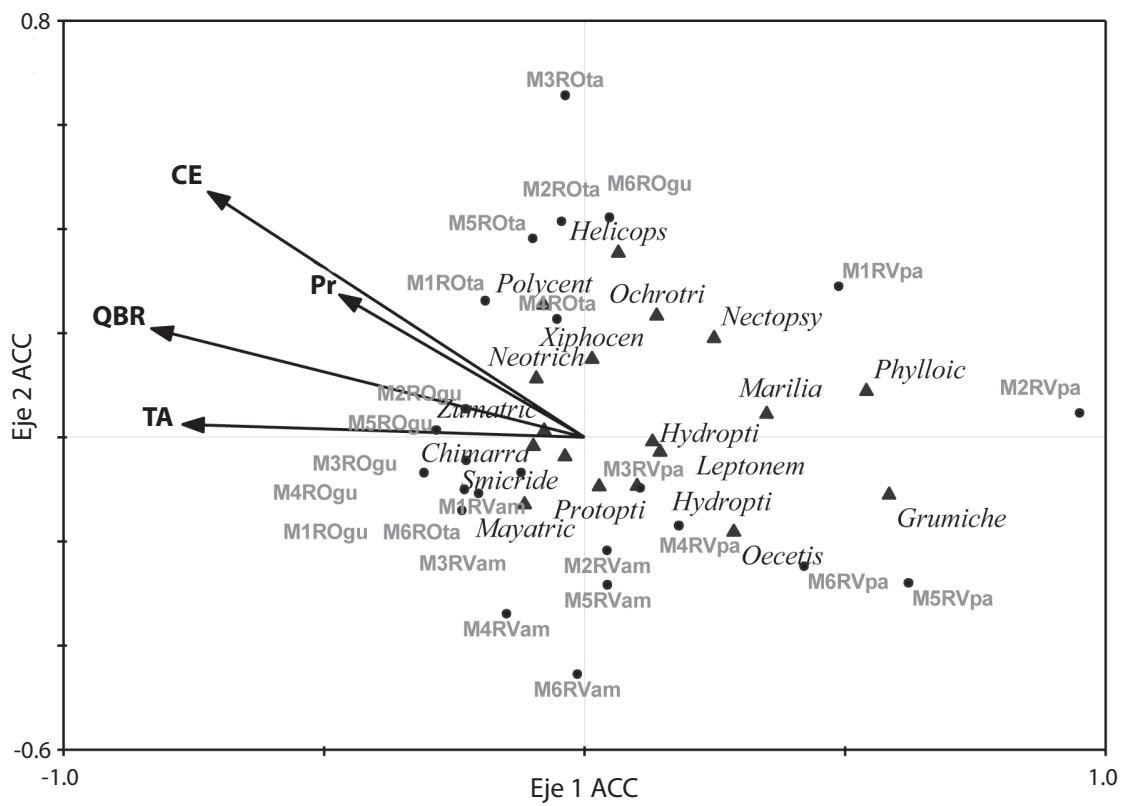

Fig. 10. Diagrama de ordenación de la comunidad de Trichoptera en los ríos Venadillo y Opia durante seis muestreos, con relación a las variables fisicoquímicas.

Fig. 10. Ordination plot of the Trichoptera assemblage in Venadillo and Opia rivers during six samplings and their relationship to physicochemical variables.

dependen de las condiciones inherentes de cada sistema acuático, del régimen de precipitación, uso del suelo y la oferta de sustratos (e.g., Urbanič, Toman \& Kruönik, 2005; Urbanič \& Toman 2007; Pastuchová, Lehotský \& Grešková, 2008).

En el presente estudio, Smicridea, Leptonema, Protoptila, Neotrichia y Chimarra, presentaron una alta dominancia que posiblemente sea el resultado de la presencia de los sustratos grava, hojarasca y roca a lo largo de los ríos Opia y Venadillo, particularmente de sustrato rocoso, el cual es dominante en varias corrientes del departamento del Tolima (Guevara et al., 2005; Reinoso et al., 2007a). Estos resultados son similares a los reportados por Huamantico \& Nessimian (2000), Bispo, Oliveira, Bini \& Sousa (2006) y Fidelis, Nessimian \& Hamada (2008), quienes indican que estos taxones pueden presentar densidades elevadas en corrientes de tercer y cuarto orden, y están frecuentemente asociados a sustratos rocosos. Por su parte, Hydroptila, Helicopsyche y
Grumichella mostraron una alta densidad en las zonas de mayor altitud en los ríos evaluados, lo que sugiere una restricción de estos organismos a la oferta y calidad de sustrato (mayor presencia de hojarasca y grava), variación del régimen de disturbio, tamaño de la corriente y/o a la menor influencia de factores antropogénicos, que afectan la estructura de los ensamblajes de insectos acuáticos (Bispo et al., 2006; Wiens, 2002). Una observación común a muchos sistemas lóticos, tanto de zonas templadas como tropicales, es que a mayor altitud predomina el sustrato hojarasca, el cual es favorecido por la mayor presencia de vegetación ribereña, menor amplitud de cauce, aguas de mejor calidad (e.g., transparentes y mejor oxigenadas), entre otras características típicas de sistemas de menor orden (Vannote, Minshall, Cummins, Sedell \& Cushing, 1980; Winemiller, Flecker \& Hoeinghaus, 2010).

Los taxones Nectopsyche, Phylloicus, Marilia y Macronema fueron poco frecuentes en las recolectas, posiblemente debido a la 
influencia de la velocidad del agua registrada en los ríos Opia y Venadillo, que no posibilitan la acumulación de material alóctono, a pesar de poseer buena calidad de la vegetación ribereña $(\mathrm{QBR}=$ bueno $)$. Se ha evidenciado que estos organismos son abundantes en corrientes de $1^{\text {er }}$ y $2^{\text {do }}$ orden en áreas con densas coberturas de vegetación asociados a cúmulos de hojarasca y bajo caudal (Bispo et al., 2006; Ríos-Touma, Prat \& Encalada, 2012).

La composición y riqueza de larvas de Trichoptera de los ríos Opia y Venadillo mostró una variación a nivel espacial, ya que en los tramos altos se registraron los taxones Hydroptila, Phylloicus, Helicopsyche y Grumichella que requieren condiciones de hábitat muy particulares, en ambientes con baja intervención antropogénica directa, mientras que en las partes bajas $(<300 \mathrm{msnm})$ se registraron aquellos más tolerantes a cambios en las condiciones fisicoquímicas del agua, tales como Smicridea, Leptonema, Protoptila y Neotrichia. La variación longitudinal en la composición, abundancia y diversidad de los ensamblajes acuáticos, particularmente de tricópteros, está regulada por diversos factores ambientales que operan a diferentes escalas espaciales que interactúan de manera compleja (Costa \& Melo, 2008; Galbraith, Vaughn \& Meier, 2008). Algunos factores como el ancho y la estructura del bosque ribereño, abertura del dosel, material de retención, vegetación acuática y sedimentos, ejercen un fuerte impacto sobre la composición taxonómica de las comunidades acuáticas, al modificar drásticamente el hábitat (Nessimian et al., 2008; Robinson \& Minshall, 1986; Zimmermann \& Death, 2002).

La presencia de una variedad de sustratos en los ecosistemas dulceacuícolas influye en la diversidad y riqueza de la fauna béntica, y en el caso de los tricópteros la arena, la hojarasca y el material particulado son sustratos que favorecen su establecimiento y colonización (Guevara et al., 2005; Vásquez-Ramos et al., 2010). Sin embargo, en nuestro estudio no se encontraron diferencias significativas en la diversidad y riqueza de taxones, a pesar de que hojarasca y arena registraron los mayores valores. En estos sustratos se destaca la dominancia de la familia Hydroptilidae (Neotrichia, Hydroptila, Leucotrichia, Zumatrichia, Mayatrichia, Ceratotrichia), la cual encuentra en ellos elementos importantes para completar el estadio larval (Guevara, 2004; López, 2007; Vásquez-Ramos et al., 2010). Nuestros resultados indican que la diversidad de tricópteros está influenciada por la heterogeneidad de sustratos en las corrientes evaluadas. Lo anterior es concordante con lo encontrado por Costa \& Melo (2008b) quienes no registraron diferencias significativas entre los microhábitats (roca, hojarasca, raíces sumergidas y musgos), en términos de riqueza de taxones. La riqueza, está principalmente asociada con la diversidad de sustratos presentes en un área determinada (Ballesteros et al., 1997; Vásquez-Ramos et al., 2010), y es a su vez influenciada por presiones antropogénicas que alteran la composición física y química del agua (Guevara et al., 2005), las cuales determinan diferentes respuestas en la colonización, frecuencia y abundancia de los tricópteros (Rincón, 1996; Posada-García \& Roldán, 2003; Muñoz-Quesada, 2004; Springer, 2006; Spies et al., 2006).

En los sistemas lóticos, diversos factores influyen directa o indirectamente a nivel longitudinal en la estructuración de las comunidades bentónicas (Guevara, 2004; Reinoso et al., 2007b; Vásquez-Ramos et al., 2010). En ríos del departamento del Tolima, se ha evidenciado que la riqueza taxonómica de los macroinvertebrados, está correlacionada con la altitud y la temperatura del agua, registrándose una mayor riqueza en las cabeceras y zonas medias de las corrientes, lo que coincide con lo reportado por otros autores (e.g., Miserendino, 2001; Vannote et al., 1980). Sin embargo, esta característica parece estar relacionada con aspectos de menor intervención antropogénica, mayor heterotrofia y abundancia de sustratos orgánicos, aguas más oxigenadas, entre otros (Clarke, Mac Nally, Bond \& Lake, 2008). No obstante, en el presente estudio realizado en un gradiente altitudinal corto, en tramos de zonas bajas ( $\sim 570-\sim 250 \mathrm{msnm})$, se registraron valores comparativamente altos de diversidad y riqueza 
de larvas de tricópteros frente a los obtenidos en estudios de otras cuencas del departamento del Tolima, donde se evalúo un mayor rango altitudinal (3 553 - 256m; Guevara et al., 2005; Vásquez-Ramos et al., 2010).

En nuestro estudio las variables fisicoquímicas mostraron una fuerte relación con el régimen de precipitación. En ambos ríos, los valores de turbidez, sólidos suspendidos y fosfatos se incrementaron en los periodos de alta lluvia y viceversa. En otras cuencas andinas, se han encontrado diferencias significativas a nivel temporal con respecto al $\mathrm{pH}$, dureza, DQO, fosfatos y nitritos, y valores de oxígeno disuelto más altos durante la temporada lluviosa que en la seca (Guevara, 2004; Guevara, Reinoso \& Villa, 2007a, 2007b; Posada et al., 2000; Rincón, 1996, 2002). Asimismo, Vásquez-Ramos et al. (2010) encontraron que el régimen pluviométrico es un factor determinante del comportamiento de las variables fisicoquímicas, ya que durante la época de lluvia estas variables muestran una mayor fluctuación, y en la época seca una mayor estabilidad.

Nuestros resultados también revelan el efecto del aumento de la precipitación, con el consecuente aumento de caudal, sobre la abundancia y riqueza de invertebrados bentónicos (e.g., Baptista, Buss, Dorville \& Nessimian, 2001, Arias-Díaz et al., 2007), atributos que se relacionan con la disponibilidad de hábitats adecuados para la colonización y establecimiento de esta fauna (Urbanič et al., 2005; Pastuchová et al., 2008). Asimismo, los aspectos de distribución y frecuencia registrados para algunos taxones en los ríos evaluados, pueden estar asociados con el hecho que los tricópteros presentan todos los grupos funcionales alimentarios (Holzenthal et al., 2007), lo que permite detectar diferencias en las densidades de ciertos grupos según el tipo de sustrato dominante (Guevara et al., 2005). No obstante, este es un tema de futuras investigaciones no solo para los ríos analizados sino para otras cuencas del departamento del Tolima y Colombia.

\section{AGRADECIMIENTOS}

Agradecemos el apoyo financiero del Comité Central de Investigaciones y al Grupo de Investigación en Zoología de la Universidad del Tolima. El primer autor agradece el apoyo a través del programa Jóvenes Investigadores e Innovadores Virginia Gutiérrez de Pineda de COLCIENCIAS, al programa de Asistentes de Docencia de la Vicerrectoría Académica de la Universidad del Tolima y a la Maestría en Ciencias Biológicas. Expresamos un agradecimiento especial a Verónica Arango, Edwin López, Carolina Gutiérrez, Angie Candia, Adriana Forero, Jaider Peña, Alexander Domínguez y Leonardo Ospina por su apoyo en campo y laboratorio. Las sugerencias de dos revisores anónimos fueron significativamente valiosas para mejorar el manuscrito original. Los errores persistentes son de nuestra responsabilidad. Esta es la contribución número 375 del Grupo de Investigación en Zoología de la Universidad del Tolima.

\section{RESUMEN}

Los ríos de bosques secos tropicales están amenazados en todo el mundo, y en Suramérica son una de las prioridades en términos de conservación. En este estudio se determinó la influencia de variables ambientales (por ejemplo precipitación) y la vegetación ribereña sobre las comunidades del orden Trichoptera en cuatro sustratos (roca, grava, arena y hojarasca) en las cuencas Opia y Venadillo (Tolima, Colombia). En cada río, en dos segmentos de $100 \mathrm{~m}$ (uno a $\sim 550$ y otro a $\sim 250 \mathrm{msnm}$ ), fueron evaluados los sustratos mencionados anteriormente. Se realizaron análisis físico-químicos, y se aplicó el índice QBR (calidad del bosque de ribera) en ambos ríos. Se recolectaron 6 282 larvas, pertenecientes a 11 familias y 22 géneros, que representan el $73.30 \%$ y $43.13 \%$ de la fauna Trichoptera registrada en Colombia, respectivamente. Las familias más abundantes fueron Hydropsychidae (49.86\%) y Philopotamidae (25.44\%). Los géneros Smicridea, Chimarra, Protoptila, Neotrichia y Leptonema fueron comunes en periodos de baja y alta precipitación. Las asociaciones de tricópteros no mostraron diferencias significativas a nivel de sustrato. Los principales factores que determinaron la composición, riqueza y abundancia de tricópteros fueron la estacionalidad y la vegetación de ribera. Sin embargo, 
las localidades situadas a mayor altitud y no urbanizadas, ofrecen mayor variedad de sustratos y mayor riqueza de géneros. Nuestros resultados indican que a futuro las larvas de Trichoptera constituyen un elemento biótico relevante en los ecosistemas dulceacuícolas, debido a que son sensibles a disturbios ambientales. Por ende, sugerimos el uso de los tricópteros para biomonitoreo en ríos tropicales. La implementación de estos estudios es urgente, teniendo en cuenta que la degradación de los ecosistemas dulceacuícolas tiende a ser intensa y persistente.

Palabras clave: insectos acuáticos, Trichoptera, bosque seco tropical, ecosistemas acuáticos, precipitación, substratos.

\section{REFERENCIAS}

Allan, D. (2004). Landscapes and riverscapes: the Influence of land use on stream ecosystems. Annual Review of Ecology, Evolution, and Systematics, 35, 257-284.

Andrade, T. E., \& Lozano, P. (1986). Sectorización hidrográfica del departamento del Tolima sector norte, Escala 1:25000. (Tesis de pregrado). Universidad del Tolima, Tolima, Colombia.

Angrisano, E. B. (1995). Insecta Trichoptera. In E. Lopretto \& G. Tell (Eds.), Ecosistemas de aguas continentales. Metodologías para su estudio (pp. 1199-1237). La Plata: Ediciones Sur.

Angrisano, E. B., \& Korob, P. G. (2001). Trichoptera. In H. R Fernández \& E. Domínguez (Eds.), Guía para la determinación de los artrópodos bentónicos Sudamericanos (pp. 55-92). Tucumán: Universidad Nacional de Tucumán.

Angrisano, E. B., \& Sganga, J. V. (2009). Trichoptera. In E. Domínguez \& H. R. Fernández (Eds.). Macroinvertebrados bentónicos sudamericanos (pp. 255-308). Tucumán: Fundación Miguel Lillo.

Arias-Díaz, D. M., Reinoso, G., Guevara, G., \& Villa, F. (2007). Distribución espacial y temporal de los coleópteros acuáticos en la cuenca del río Coello (Tolima, Colombia). Caldasia, 29(1), 177-194.

Ballesteros, Y. V., Zúñiga, M. C., \& Rojas de Hernández, A. M. (1997). Distribution and structure of the order Trichoptera in various drainages of the Cauca River basin, Colombia, and their relationship to water quality. In Proceedings of the 8th International Symposium on Trichoptera (pp. 19-23). Ohio: Ohio Biological Survey.

Baptista, D. F., Buss, D. F., Dorville L. F. M., \& Nessimian, J. L. (2001). Spatial and temporal organization of aquatic insects assemblages in the longitudinal gradient of a tropical river. Revista Brasileira de Biologia, 61(2), 295-304.
Barreda-Bautista, B. de la, López-Caloca, A., Couturier, S., \& Silván-Cárdenas, J. S. (2002). Tropical dry forests in the global picture: the challenge of remote sensingbased change detection in tropical dry environments. In E. Carayannis (Ed.), Planet Earth 2011 - Global warming challenges and opportunities for policy and practice (pp. 646). Wallingford: InTech. Recuperado de http://www.intechopen.com/source/pdfs/21119/ InTechTropical_dry_forests_in_the_global_

picture_the_challenge_of_remote_sensing_based_change detection_in_tropical_dry_environments.pdf

Beisel, J. N., Usseglio-Polatera, P., Thomas, S., \& Moreteau J. S. (1998). Stream community structure in relation to spatial variation: the influence of microhabitat characteristics. Hydrobiologia, 389, 73-88.

Bianchi, C. A., \& Haig, S. M. (2013). Deforestation Trends of Tropical Dry Forests in Central Brazil. Biotropica, 45(3), 395-400.

Bispo, P. C., Oliveira, L. G., Bini, L. M., \& Sousa, K. G. (2006). Ephemeroptera, Plecoptera and Trichoptera assemblages from riffles in mountain streams of Central Brazil: environmental factors influencing the distribution and abundance of inmatures. Brazilian Journal of Biology, 66(2B), 611-622.

Castañeda, A., Medina, N., Méndez, A., \& Quimbayo, F. (1989). Estudio morfométrico e hidroclimático de la subcuenca del río Opia, departamento del Tolima. (Tesis de pregrado). Universidad del Tolima, Tolima, Colombia.

Chará, J., Pedraza, G., Giraldo, L., \& Hincapié, D. (2007). Efecto de los corredores ribereños sobre el estado de quebradas en la zona ganadera del río La Vieja, Colombia. Agroforestería en las Américas, 45, 72-78.

Chazdon, R. (2003). Tropical forest recovery: legacies of human impact and natural disturbances. Perspectives in Plant Ecology, Evolution and Systematics, 6, 51-71.

Clarke, K. R., \& Gorley, R. N. (2006). PRIMER v6: User Manual/Tutorial. PRIMER-E, Plymouth.

Clarke, A., Mac Nally, R., Bond, N., \& Lake, P. S. (2008). Macroinvertebrate diversity in headwater streams: a review. Freshwater Biology, 53(9), 1707-1721.

Colwell, S. R., \& Hix, D. M. (2008). Adaptation of the QBR index for use in riparian forests of Central Ohio. Proceedings 16th Central Hardwood Forest Conference, 16, 331-340.

CORTOLIMA. (1998). Corporación Autónoma Regional del Tolima. Plan de gestión ambiental para el departamento del Tolima 1998-2002. Ibagué, Colombia.

Costa, S. S., \& Melo, A. S. (2008). Beta diversity in stream macroinvertebrate assemblages: among-site and among-microhabitat components. Hydrobiologia, 598(1), 131-138. 
Death, R. G., \& Collier, K. J. (2009). Measuring stream macroinvertebrate responses to gradients of vegetation cover: when is enough enough? Freshwater Biology, 55(7), 1447-1464.

Díaz, J. M. (2006). Bosque seco tropical Colombia. Cali: Banco de Occidente.

Domínguez, E., \& Fernández, H. R. (2009). Macroinvertebrados bentónicos sudamericanos. Sistemática y biología. Tucumán: Fudanción Miguel Lillo.

Dudgeon, D., Arthington, A. H., Gessner, M. O., Kawabata, Z. I., Knowler, D. J., Lévêque, C., Naiman, R. J., Prieur-Richard, A. H., Soto, D., Stiassny, M. L. J., \& Sullivan, C. A. (2006). Freshwater biodiversity: importance, threats, status and conservation challenges. Biological reviews of the Cambridge Philosophical Society, 81(2), 163-82.

Dunn, R. R. (2004). Recovery of faunal communities during tropical forest regeneration. Conservation Biology, 18, 302-309.

Encalada, A. C., Calles, J., Ferreira, V., Canhoto, C. M., \& Graça, M. A. S. (2010). Riparian land use and the relationship between the benthos and litter decomposition in tropical montane streams. Freshwater Biology, 55, 1719-1733.

Fidelis, L., Nessimian, J. L., \& Hamada, N. (2008). Distribuição espacial de insetos aquáticos em igarapés de pequena ordem na Amazônia Central. Acta Amazonica, 38(1), 127-134.

Galbraith, H. S., Vaughn, C. C., \& Meier, C. K. (2008). Environmental variables interact across spatial scales to structure trichopteran assemblages in Ouachita Mountain rivers. Hydrobiologia, 596, 401-411.

Guevara, G. (2004). Análisis faunístico del orden Trichoptera en su estado larval en la cuenca del río Coello, departamento del Tolima. (Tesis de Maestria). Universidad del Tolima, Tolima, Colombia.

Guevara, G., Reinoso, G., \& Villa, F. (2005). Estudio del orden Trichoptera en su estado larval en la cuenca del río Coello Departamento del Tolima. Revista de la Asociación Colombiana de Ciencias Biológicas, 17, 59-70.

Guevara, G., Reinoso G., \& Villa, F. (2007a). Caddisfly larvae (Insecta: Trichoptera) of the Coello River basin in Central Colombia. Trichopteron, 24, 8-12.

Guevara, G., Reinoso G., \& Villa, F. (2007b). Caddisfly larvae (Insecta: Trichoptera) of the Coello River basin in Tolima (Colombia): Spatial and temporal patterns and bioecological aspects. In J. Bueno-Soria, R. Narba-Alvarez \& B. Armitage (Eds.), Proceedings of the XIIth International Symposium on Trichoptera (pp. 8-12). Columbus: The Caddis Press.

Guevara, G., Reinoso, G., García, J. E., Franco, L. M., García, L. J., Yara, D. C., Negover, G., Ocampo, M. L., Quintana, M. I., Pava, D. Y., Flórez, N. Y., Ávila,
M. F., Hernández, E. E., Lozano, L. A., Guapucal, M., Borrero, D. A., \& Olaya, E. J. (2008). Aportes para el análisis de ecosistemas fluviales: una visión desde ambientes ribereños. Revista Tumbaga, 3, 109-127.

Hammer, O., Harper, D. A. T., \& Ryan, P. D. (2001). PAST: Paleontological Statistics software package for education and data analysis. Palaeontología Electrónica, 4(1), 9 .

Holdridge, L. R. (1987). Ecología basada en zonas de vida. San José: Instituto Interamericano de Cooperación para la Agricultura.

Holzenthal, R. W., Blahnik, R. J., Prather, A. L., \& Kjer, K. M. (2007). Order Trichoptera Kirby, 1813 (Insecta), Caddisflies. Zootaxa, 1668, 639-698.

Huamantico, A. A., \& Nessimian, J. L. (2000). Variation and life strategies of the Trichoptera (Insecta) larvae community in a first order tributary of the Paquequer River, southeastern Brazil. Revista Brasileira de Biologia, 60(1), 73-82.

IAvH. (1998). El bosque seco tropical (Bs-T) en Colombia. Bogotá: Instituto de Investigación de Recursos Biológicos Alexander von Humboldt.

Jáimez-Cuéllar, P., Vivas, S., Bonada, N., Robles, S., Mellado, A., Álvarez, M., Áviles, J., Casas, J., Ortega, M., Pardo, I., Prat, N., Rieradevall, M., SáinzCantero, C. E., Sánchez-Ortega, A., Suárez, M. L., Toro, M., Vidal-Abarca, M. R., Zamora-Muñoz, C. \& Alba-tercedor, J. (2002). Protocolo GUADALMED (PRECE). Limnetica, 21(3-4), 187-204.

Janzen, D. H. (1988). Tropical dry forest. In E. O. Wilson (Ed.), Biodiversity (pp. 538). Washington: National Academy of Sciences/Smithsonian Institution.

Johnson, L. B., \& Host, G. E. (2010). Recent developments in landscape approaches for the study of aquatic ecosystems. Freshwater Biology, 29, 41-66.

Latrubesse, E. M., Stevaux, J. C., \& Sinha, R. (2005). Tropical rivers. Geomorphology, 70(3-4), 187-206.

Leira, M., \& Sabater, S. (2005). Diatom assemblages distribution in catalan rivers, NE Spain, in relation to chemical and physiographical factors. Water Research, $39,73-82$

López, E. (2007). Análisis faunístico de las larvas del orden Trichoptera en la cuenca del río Prado y la subcuenca de Amoyá (Tolima-Colombia). (Tesis de pregrado). Universidad del Tolima, Tolima, Colombia.

Miserendino, M. L. (2001). Macroinvertebrate assemblages in Andean Patagonian rivers and streams: environmental relationships. Hydrobiologia, 444, 147-158.

Motta, R. L., \& Uieda, V. S. (2004). Diet and trophic groups of an aquatic insect community in a tropical stream. Brazilian Journal of Biology, 64, 809-17. 
Munné, A., Prat, N., Solà, C., Bonada, N., \& Rieradevall, M. (2003). A simple field method for assessing the ecological quality of riparian habitat in rivers and streams: QBR index. Aquatic Conservation: Marine and Freshwater Ecosystems, 13(2), 147-163.

Muñoz-Quesada, F. (2004). El orden Trichoptera (Insecta) en Colombia, II: inmaduros y adultos, consideraciones generales. In F. Fernández, M. Andrade \& G. Amat (Eds.). Insectos de Colombia. Vol. III (pp. 319334). Bogotá: Universidad Nacional de ColombiaInstituto Humboldt.

Murphy, P., \& Lugo, A. (1986). Ecology of tropical dry forest. Annual review of ecology and systematics, 17, 67-88.

Naiman, R., Decamps, H., \& Pollock, M. (2005). Riparia: Ecology, conservation, and management of streamside communities. Amsterdam: Elsevier Academic Press.

Naiman, R. J., Decamps, H., \& Pollock, M. (1993). The Role of Riparian Corridors in Maintaining Regional Biodiversity. Ecological Applications, 3(2), 209-212.

Negi, M., \& Singh., H. R (1990). Substratum as determining factor for bottom fauna in River Alaknanda. Proceeding of the National Academy of Science India, 56, 417-423.

Nessimian, J. L., Venticinque, E. M., Zuanon, J., Marco, P., Gordo, M., Fidelis, L., Batista, J. D., \& Juen, L. (2008). Land use, habitat integrity, and aquatic insect assemblages in Central Amazonian streams. Hydrobiologia, 614(1), 117-131.

Oliveira, A. L. H., \& Nessimian, J. L. (2010). Spatial distribution and functional feeding groups of aquatic insect communities in Serra da Bocaina streams, southeastern Brazil. Acta Limnologica Brasiliensia, $22(4), 424-441$.

Oliveira, A. M., Hamada, N., \& Nessimian, J. L. (2005). Chaves de identificação de larvas para famílias e gêneros de Trichoptera (Insecta) da Amazônia central, Brasil. Revista Brasileira de Entomologia, 49(2), $181-204$

Oliveira, A. M., \& Hamada, N. (2004). Ceratotrichia Flint, 1992 (Trichoptera: Hydroptilidae) larval and pupal description and new genus records for Brazil. Entomotropica, 19(1), 31-37.

Palacios-Vargas J. G., Castaño-Meneses, G., GómezAnaya, J. A., Martínez-Yrizar, A., Mejía-Recamier, B.E., \& Martínez-Sánchez, J. (2007). Litter and soil arthropods diversity and density in a tropical dry forest ecosystem in Western Mexico. Biodiversity and Conservation, 16, 3703-3717.

Pardo, I., \& Armitage, P. D. (1997). Species assemblages as descriptors of mesohabitats. Hydrobiologia, 344, 111-128.
Pastuchová, Z., Lehotský M., \& Grešková, A. (2008). Influence of morphohydraulic habitat structure on invertebrate communities (Ephemeroptera, Plecoptera and Trichoptera). Biologia, 63(5), 720-729.

Poff, N. L., \& Zimmerman, J. K. H. (2010). Ecological responses to altered flow regimes: a literature review to inform the science and management of environmental flows. Freshwater Biology, 55(1), 194-205.

Posada, J., Roldán, G., \& Ramírez, J. (2000). Caracterización fisicoquímica y biológica de la calidad de aguas de la cuenca de la quebrada Piedras Blancas, Antioquia, Colombia. Revista de Biologia Tropical, 48(1), 59-70.

Posada-Garcia, J. A., \& Roldán, G. (2003). Clave ilustrada y diversidad de las larvas de Trichoptera en el Noroccidente de Colombia. Caldasia, 25(1), 169-192.

Quesada, M., Sanchez-Azofeifa, G. A., Alvarez-Añorve, M., Stoner, K. E., Avila-Cabadilla, L., Calvo-Alvarado, J., Castillo, A., Espíritu-Santo, M. M., Fagundes, M., Fernándes, G. W., Gamón, J., Lopezaraiza-Mikel, M., Lawrence, D., Morellato, L. P. C., Powers, J. S., Neves, F. S., Rosas-Guerrero, V., Sayago, R., \& Sanchez-Montoya, G. (2009). Succession and management of tropical dry forests in the Americas: Review and new perspectives. Forest Ecology and Management, 258, 1014-1024.

Reinoso, G., Guevara, G., Arias, D., \& Villa, F. (2007a). Aspectos bioecológicos de la fauna entomológica de la cuenca mayor del rio Coello-departamento del Tolima. Revista de la Asociación Colombiana de Ciencias Biológicas, 19, 65-71.

Reinoso, G., Guevara, G., Vejarano, M., Garcia, J., \& Villa, F. (2007b). Evaluación del río Prado a partir de los macroinvertebrados y de la calidad del agua. Revista de la Asociación Colombiana de Ciencias Biológicas, 19, 141-154.

Rincón, M. E. (1996). Aspectos bioecológicos de los tricópteros de la quebrada Carrizal (Boyacá, Colombia). Revista Colombiana de Entomología, 22(1), 53-60.

Rincón, M. E. (2002). Comunidad de insectos acuáticos de la quebrada Mamarramos (Boyacá, Colombia). Revista Colombiana de Entomología, 28(1), 101-108.

Ríos-Touma, B., Prat, N., \& Encalada, A. C. (2012). Invertebrate drift and colonization processes in a tropical Andean stream. Aquatic Biology, 14, 233-246.

Robinson, C. T., \& Minshall, G. W. (1986). Effects of disturbance frequency on stream benthic community structure in relation to canopy cover and season. Journal of the North American Benthological Society, 5(3), 237-248.

Roldán, G., \& Ramírez, J. J. (2008). Fundamentos de limnología neotropical ( $2^{\mathrm{a}}$ ed.). Antioquia: Editorial Universidad de Antioquia. 
Spies, M. R., Froehlich, C. G., \& Kotzian, C. B. (2006) Composition and diversity of Trichoptera (Insecta) larvae communities in the middle section of the Jacuí river and some tributaries, State of Rio Grande do Sul, Brazil. Iheringia Série Zoologia, 96(4), 389-398.

Springer, M. (2006). Clave taxonómica para larvas de las familias del orden Trichoptera (Insecta) de Costa Rica. Revista de Biologia Tropical, 54(1), 273-286.

Strayer, D. L. (2006). Challenges for freshwater invertebrate conservation. Journal of the North American Benthological Society, 25(2), 271-287.

Subramanian, K. A., \& Sivaramakrishnan, K. G. (2005). Habitat and microhabitat distribution of stream insect communities of the Western Ghats. Current Science, 89(6), 976-987.

Trejo, I., \& Dirzo, R. (2000). Deforestation of seasonally dry tropical forest: a national and local analysis in Mexico. Biological Conservation, 94, 133-142.

Urbanič, G., Toman, M. J., \& Kruönik, C. (2005). Microhabitat type selection of caddisfly larvae (Insecta: Trichoptera) in a shallow lowland stream. Hydrobiologia, 541, 1-12.

Urbanič, G., \& Toman M. J. (2007). Influence of environmental variables on stream caddis larvae in three Slovenian ecoregions: Alps, Dinaric Western Balkansa and Pannonian Lowland. International Review of Hydrobiology, 92(4-5), 582-602.

ter Braak, C. J. F., \& Smilauer, P. (2009). Canoco for Windows (version 4.56): A FORTRAN Program for Canonical Community Ordination. Wageningen: Centre for Biometry.
Utz, R. M., Hilderbrand, R. H., \& Boward, D. M. (2009). Identifying regional differences in threshold responses of aquatic invertebrates to land cover gradients. Ecological Indicators, 9(3), 556-567.

Vannote, R. L., Minshall, G. W., Cummins, K. W., Sedell, J. R., \& Cushing, C. E. (1980). The River Continuum Concept. Canadian Journal of Fisheries and Aquatic Sciences, 37(1), 130-137.

Vásquez-Ramos, J. M., Ramírez-Díaz, F., Reinoso, G., \& Guevara, G. (2010). Distribución espacial y temporal de los tricópteros inmaduros en la cuenca del rio Totare (Tolima-Colombia). Caldasia, 32(1), 129-148.

Vásquez-Ramos, J. M., \& Reinoso, G. (2012). Estructura de la fauna béntica en corrientes de los Andes colombianos. Revista Colombiana de Entomología, 38(2), 351-358.

Wiens, J. A. (2002). Riverine landscapes: taking landscape ecology into the water. Freshwater Biology, 47(4), 501-515.

Winemiller, K. O., Flecker, A. S., \& Hoeinghaus, D. J. (2010). Patch dynamics and environmental heterogeneity in lotic ecosystems. Society, 29, 84-99.

Zimmermann, E. M., \& Death, R. G. (2002). Effect of substrate stability and canopy cover on stream invertebrate communities. New Zealand Journal of Marine and Freshwater Research, 36(3), 537-545.

Zúñiga, M. C., Rojas, A., \& Serrato, C. (1994). Interrelación de indicadores ambientales de calidad en cuerpos de agua superficiales del Valle del Cauca. Revista Colombiana de Entomología, 20(2), 124-130. 